\title{
Doppler-Streak Attenuation via Oscillatory-plus-Transient Decomposition of IQ Data
}

\author{
Ivan W. Selesnick ${ }^{1,2}$, Ke Yong Li ${ }^{2}$, S. Unnikrishna Pillai ${ }^{1,2}$, Braham Himed ${ }^{3}$ \\ ${ }^{1}$ Polytechnic Institute of New York University, Brooklyn, NY 07040 \\ ${ }^{2}$ C \& P Technologies Inc., Closter, NJ 07624 \\ ${ }^{3}$ WPAFB, AFRL/RYRT, Dayton, OH, 45433
}

Keywords: Range-Doppler processing, sparse signal representation, interference suppression, signal separation.

\begin{abstract}
In the formation of range-Doppler images, transient interference can result in Doppler-streak artifacts. These Doppler-streaks hinder the utility of the range-Doppler image and may obscure moving targets of interest. This paper describes a method for pre-processing the IQ data to substantially attenuate Doppler streaks in the subsequently formed range-Doppler images. The method consists of decomposing the IQ data for each range bin into the sum of an oscillatory component and a transient component.
\end{abstract}

\section{Introduction}

In the formation of range-Doppler profile images, conventional Doppler processing can lead to streaking artifacts, such as those clearly visible in Fig. $1 \mathrm{a}^{1}$. From the range-Doppler profile itself, the underlying reason for these streaks is unclear. There are several potential causes: (1) A moving target with a fast changing velocity — if a target accelerates during the dwell, the energy will be spread with respect to Doppler. (2) Persistent (quasi-stationary) wide-band interference. (3) A transient event (or brief interference) in the IQ data - a strong interference present for only a few pulses will be generate a wide-band Doppler spectrum. From the range-Doppler profile itself, a Dopplerstreak could indicate any one of these possibilities. In order to distinguish the cause, it is informative to inspect the IQ data itself.

For the range-Doppler profile illustrated in Fig. 1a, inspection of the IQ data reveals that the Doppler-streaks are due to transient interference in the IQ data. If it can be presumed that a target will be present for all or most of the pulses in the coherent pulse interval, then it can be concluded that the transient in Fig. 1a, which lasts for only a few of the 256 pulses, is not due to a target of interest.

This paper describes a pre-processing technique that substantially attenuates Doppler streaking due to transient in-

This work was supported by the Air Force Research Laboratory (AFRL) Sensors Directorate, Radar Signal Processing Branch under SBIR contract FA8650-09-C-1628.

Approved for public release, distribution unlimited.

${ }^{1}$ This data used to generate this result was collected from Jan. 9, 2011 to Jan. 11, 2011 under the TRRI program [6] and provided under the aforementioned SBIR contract. terference. It is proposed that the IQ data be first separated into two components (an oscillatory component and a transient component), and second, that the range-Doppler profile be generated using only the oscillatory component.

\section{Oscillatory-Transient Decomposition}

The proposed pre-processing approach utilizes a nonlinear algorithm that decomposes a given signal into the sum of an oscillatory signal and a transient signal $[3,4,7,9]$. That is, given a one-dimensional signal $\mathbf{x}$, the algorithm determines $\mathbf{x}_{1}$ and $\mathbf{x}_{2}$ such that

$$
\mathbf{x}=\mathbf{x}_{1}+\mathbf{x}_{2}
$$

where $\mathbf{x}_{1}$ has an oscillatory behavior and $\mathbf{x}_{2}$ has a transient behavior. The oscillatory signal $\mathbf{x}_{1}$ need not be narrow band nor need the frequencies of oscillation be known. Further, the transient signal $\mathbf{x}_{2}$ need not follow a pre-determined waveform template; it may have some unspecified shape. In addition, $\mathbf{x}_{1}$ and $\mathbf{x}_{2}$ may overlap in time and frequency; therefore, conventional methods of time-frequency selective filtering will be of limited use. However, non-linear methods based on the optimization of suitably defined nonquadratic cost functions can be used to achieve this type of separation $[7,9,10]$. The method applies equally well to purely real and general complex data.

It is shown below that the oscillatory-plus-transient decomposition (1) can be used to avoid the Doppler-streaking artifacts visible in the range-Doppler profile, for example in Fig. 1a. In particular, the IQ data can be pre-processed using the decomposition in order to separate the IQ data into oscillatory and transient components. In this way, the transient interference (if any) can be (partially) removed from the IQ data. Conventional Doppler processing (taper+FFT) can then be applied to the oscillatory component of the IQ data. The result of applying this pre-Doppler processing procedure is illustrated in Fig. 1b. Note that in Fig. 1b, numerous Doppler-streaks that are present in Fig. 1a have been successfully removed. Moreover, the procedure does not appear to adversely affect the features and potential targets of interest.

\section{Example}

To elucidate the method, it is informative to inspect the original IQ data to clarify the cause for the Doppler-streak. Consider the Doppler-streak at range bin 45 in Fig. 1a; the 


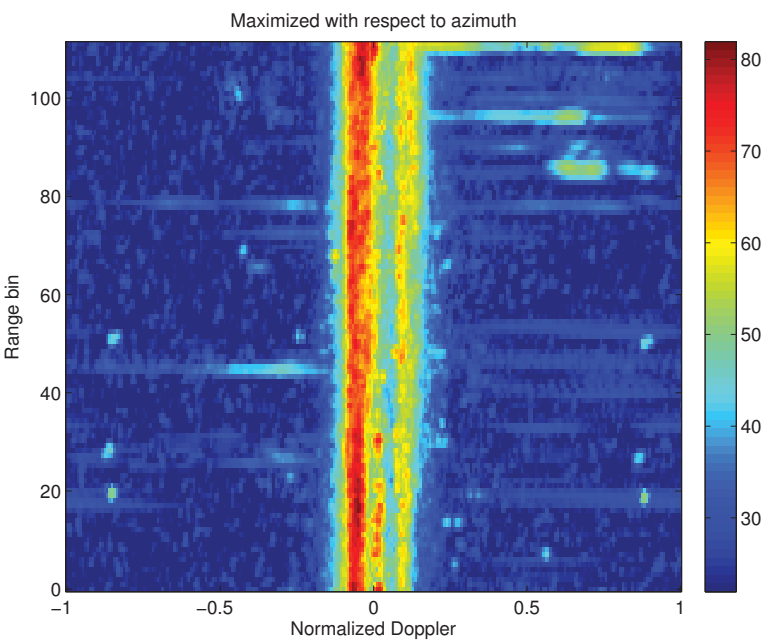

(a) Without preprocessing

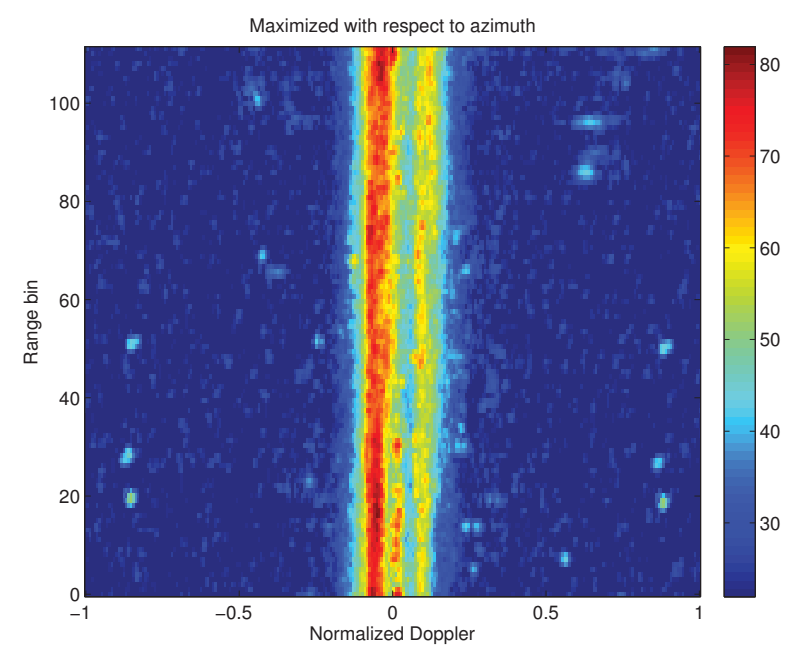

(b) With preprocessing

Figure 1: Range-Doppler profile. Note the presence of Doppler-streaks in (a) due to high-power transients present in the IQ data. After pre-processing the IQ data, the Doppler-streak artifacts are substantially attenuated (b).

IQ data, corresponding to this range, is shown in Fig. 2a. Clearly visible in the IQ data is a transient event around pulse 115. This transient event is the cause of the Dopplerstreak in the range-Doppler profile. When the oscillatoryplus-transient decomposition algorithm is applied to this IQ data, the signals illustrated in Figs. $2 b$ and $2 c$ are obtained. The sum of the oscillatory and transient signals is exactly equal to the IQ signal shown in Fig. 2a. It should be noted that this decomposition is achieved by the algorithm with no prior knowledge of where the transient event is located in the signal. The algorithm is not based on any explicit segmentation of the IQ signal; instead the oscillatory and transient components are found jointly.

The effect of the oscillatory-plus-transient decomposition on the IQ data can be further analyzed by inspecting the spectra of the IQ data and of the oscillatory and transient components calculated as the result of the algorithm. To that end, Fig. 3a illustrates the spectrum of the original IQ

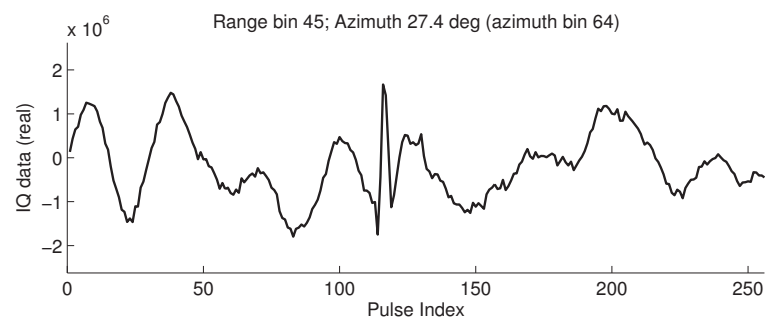

(a) IQ data

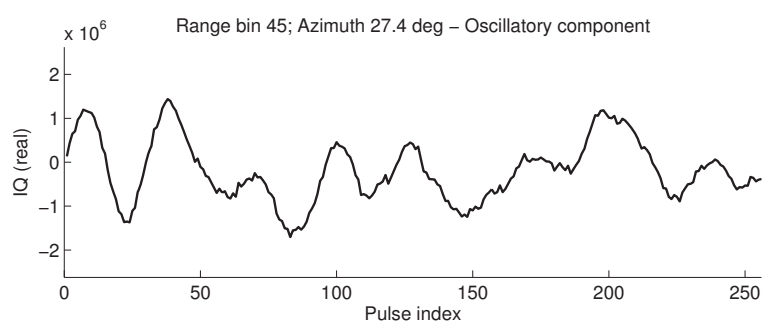

(b) Calculated oscillatory component

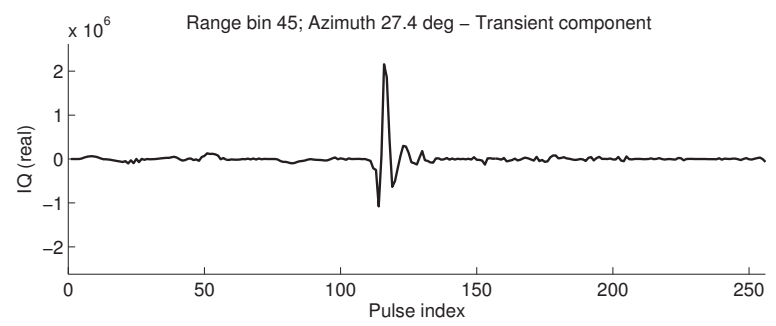

(c) Calculated transient component

Figure 2: IQ data for range bin 45 and the calculated oscillatory and transient components. The transient observed in the IQ data around pulse index 115 explains the Dopplerstreaks visible in Figs.1a. The transient and oscillatory components have been well separated. (The figure illustrates only the real part of the complex IQ data.)

data (shown in Fig. 2a). The transient causes a broad spectral peak at a normalized Doppler of about -0.3 . This broad spectral peak constitutes the Doppler streak in Fig. 1a. Figure $3 \mathrm{~b}$ illustrates the spectrum of the oscillatory component (shown in Fig. 2b); because the transient has been eliminated, the spectrum is free of the broad spectral peak and hence the subsequently formed range-Doppler image is free of the Doppler streak. Figure 3c illustrates the spectrum of the transient component which consists essentially of only the broad peak.

Note in Fig. 3 that the spectra of the two components overlap, yet the algorithm achieves an effective separation nevertheless.

\section{Algorithm}

The oscillatory-plus-transient decomposition algorithm is based on the minimization of a convex (but non-quadratic and non-differentiable) cost function.

The algorithm assumes the existence of two (possibly overcomplete) transforms $\Phi_{1}$ and $\Phi_{2}$ with which sparse representations of the two components can be obtained respec- 


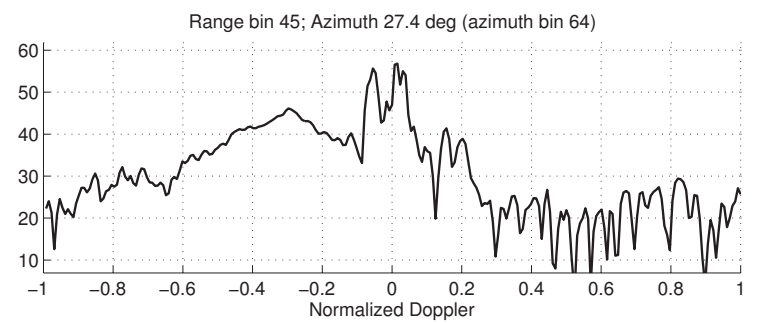

(a) Spectrum of IQ data

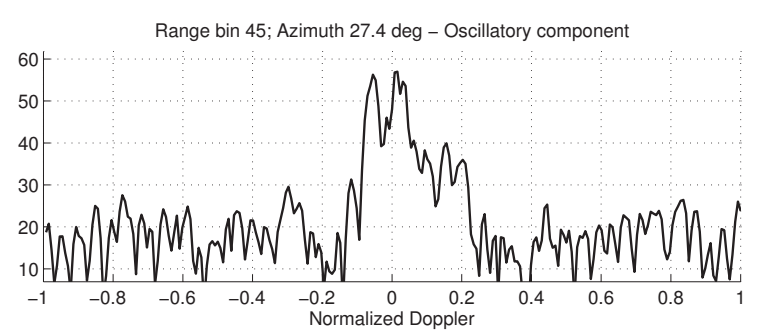

(b) Spectrum of calculated oscillatory component

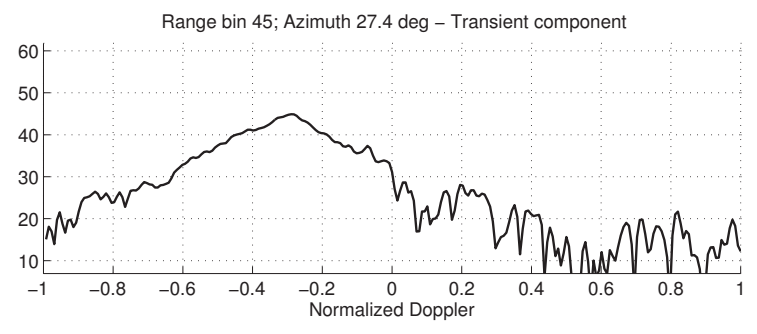

(c) Spectrum of calculated transient component

Figure 3: The frequency spectra of the IQ data and calculated components illustrated in Fig. 2.

tively. In this work, $\Phi_{i}$ are short-time Fourier transforms with different window lengths. Note that the transform coefficients used to represent the signal $\mathrm{x}$ are not unique. The algorithm assumes that $\mathrm{x}$ can be written as

$$
\mathbf{x}=\Phi_{1}^{*} \mathbf{a}_{1}+\Phi_{2}^{*} \mathbf{a}_{2}
$$

where $\mathbf{a}_{1}$ and $\mathbf{a}_{2}$ are sparse vectors. One approach to compute an (approximately) sparse representation is by minimizing the $\ell_{1}$ norms of the vectors $\mathbf{a}_{1}$ and $\mathbf{a}_{2}$. Specifically, the optimization problem is:

$$
\begin{array}{ll}
\underset{\mathbf{a}_{1}, \mathbf{a}_{2}}{\operatorname{argmin}} & \theta\left\|\mathbf{a}_{1}\right\|_{1}+(1-\theta)\left\|\mathbf{a}_{2}\right\|_{1} \\
\text { such that } & \mathbf{x}=\Phi_{1}^{*} \mathbf{a}_{1}+\Phi_{2}^{*} \mathbf{a}_{2} .
\end{array}
$$

The parameter $\theta$ is a scalar with $0<\theta<1$. Nominally, $\theta$ can be set to 0.5 , however, in practice $\theta$ can be slightly adjusted so as to obtain a more favorable decomposition. Once $\mathbf{a}_{1}$ and $\mathbf{a}_{2}$ are obtained, the calculated components are given by

$$
\mathbf{x}_{1}=\Phi_{1}^{*} \mathbf{a}_{1}, \quad \mathbf{x}_{2}=\Phi_{2}^{*} \mathbf{a}_{2} .
$$

The idea of using sparse signal representation/approximation as a means to perform signal separation is developed in $[5,10]$.
The constrained optimization problem (2) can be solved using any of several iterative algorithms. Based on the recent 'split-variable augmented Lagrangian shrinkage algorithm' (SALSA) [1], a simple iterative algorithm for solving (2) using the alternating direction method of multipliers (ADMM) is derived in Appendix B. For oscillatory-plustransient signal decomposition, see also [8, 7, 9] and references therein. For further details regarding ADMM, see [2].

\subsection{Inapplicability of Least Squares}

It is interesting to consider a modification of the cost function (2), where the energy of $\mathbf{a}_{i}$ is used in place of the $\ell_{1}$ norm:

$$
\begin{array}{ll}
\underset{\mathbf{a}_{1}, \mathbf{a}_{2}}{\operatorname{argmin}} & \theta\left\|\mathbf{a}_{1}\right\|_{2}^{2}+(1-\theta)\left\|\mathbf{a}_{2}\right\|_{2}^{2} \\
\text { such that } & \mathbf{x}=\Phi_{1}^{*} \mathbf{a}_{1}+\Phi_{2}^{*} \mathbf{a}_{2} .
\end{array}
$$

Does this optimization problem also yield a separation of the signal $\mathbf{x}$ into two distinct components? The optimization problem (3) can be solved in closed form. As discussed in [7], using (3), the solution is given by

$$
\hat{\mathbf{x}}_{1}=(1-\theta) \mathbf{x}, \quad \hat{\mathbf{x}}_{2}=\theta \mathbf{x},
$$

which provides absolutely no useful signal separation. Both components are just scaled versions of the mixed signal $\mathbf{x}$. Therefore, the $\ell_{1}$ norm (or other sparsity inducing penalty function) in (2) is essential for this approach to be effective. This is an example of the use of sparsity to solve a problem which does not have an analog in least squares signal processing.

\section{A Conclusion}

The paper describes the suppression of transient interference in IQ data for the purpose of improving range-Doppler images and subsequent processing. The algorithm does not require that the transients be explicitly identified, nor does the algorithm require any exlicit segmentation. The method is based on sparse signal representation using two simultaneous short-time Fourier transforms implemented in parallel on the IQ data using short and long windows (frames). The algorithm is able to reduce the Doppler smearing evident in the range-Doppler profile.

\section{B Derivation of algorithm}

Consider the constrained minimization problem:

$$
\begin{array}{ll}
\underset{\mathbf{a}_{1}, \mathbf{a}_{2}}{\operatorname{argmin}} & \lambda_{1}\left\|\mathbf{a}_{1}\right\|_{1}+\lambda_{2}\left\|\mathbf{a}_{2}\right\|_{1} \\
\text { such that } & \mathbf{x}=\Phi_{1}^{*} \mathbf{a}_{1}+\Phi_{2}^{*} \mathbf{a}_{2},
\end{array}
$$

where $\lambda_{1}$ and $\lambda_{2}$ are $\theta$ and $(1-\theta)$ in (2). We assume

$$
\Phi_{i}^{*} \Phi_{i}=\mathbf{I}, \quad i=1,2 .
$$


The first step is to apply variable splitting:

$$
\begin{aligned}
\underset{\mathbf{a}_{1}, \mathbf{a}_{2}, \mathbf{u}_{1}, \mathbf{u}_{2}}{\operatorname{argmin}} & \lambda_{1}\left\|\mathbf{u}_{1}\right\|_{1}+\lambda_{2}\left\|\mathbf{u}_{2}\right\|_{1} \\
\text { such that } & \mathbf{x}=\Phi_{1}^{*} \mathbf{a}_{1}+\Phi_{2}^{*} \mathbf{a}_{2} \\
& \mathbf{u}_{1}=\mathbf{a}_{1} \\
& \mathbf{u}_{2}=\mathbf{a}_{2} .
\end{aligned}
$$

Now ADMM can be applied to obtain the algorithm:

$$
\begin{aligned}
& \mathbf{u}_{1}, \mathbf{u}_{2} \leftarrow \underset{\mathbf{u}_{1}, \mathbf{u}_{2}}{\operatorname{argmin}}\left\{\begin{array}{l}
\lambda_{1}\left\|\mathbf{u}_{1}\right\|_{1}+\lambda_{2}\left\|\mathbf{u}_{2}\right\|_{2} \\
+\mu_{1}\left\|\mathbf{u}_{1}-\mathbf{a}_{1}-\mathbf{d}_{1}\right\|_{2}^{2} \\
+\mu_{2}\left\|\mathbf{u}_{2}-\mathbf{a}_{2}-\mathbf{d}_{2}\right\|_{2}^{2}
\end{array}\right. \\
& \mathbf{a}_{1}, \mathbf{a}_{2} \leftarrow\left\{\begin{array}{l}
\underset{\mathbf{a}_{1}, \mathbf{a}_{2}}{\operatorname{argmin}}\left\{\begin{array}{l}
\mu_{1}\left\|\mathbf{u}_{1}-\mathbf{a}_{1}-\mathbf{d}_{1}\right\|_{2}^{2} \\
+ \\
\mu_{2}\left\|\mathbf{u}_{2}-\mathbf{a}_{2}-\mathbf{d}_{2}\right\|_{2}^{2}
\end{array}\right. \\
\text { such that } \quad \mathbf{x}=\Phi_{1}^{*} \mathbf{a}_{1}+\Phi_{2}^{*} \mathbf{a}_{2}
\end{array}\right. \\
& \mathbf{d}_{1} \leftarrow \mathbf{d}_{1}-\left(\mathbf{u}_{1}-\mathbf{a}_{1}\right) \\
& \mathbf{d}_{2} \leftarrow \mathbf{d}_{2}-\left(\mathbf{u}_{2}-\mathbf{a}_{2}\right) \\
& \text { go to (7a). }
\end{aligned}
$$

The parameters $\mu_{i}$ should be positive scalars chosen by the user. The value of $\mu_{i}$ will not affect the solution to which the algorithm converges, but will affect the rate of convergence.

Note that $\mathbf{u}_{1}$ and $\mathbf{u}_{2}$ are decoupled in (7a), so one can write

$$
\mathbf{u}_{i} \leftarrow \operatorname{argmin} \lambda_{i}\left\|\mathbf{u}_{i}\right\|_{1}+\mu_{i}\left\|\mathbf{u}_{i}-\mathbf{a}_{i}-\mathbf{d}_{i}\right\|_{2}^{2},
$$

for $i=1,2$, for which the solution is given explicitly in terms of the soft-threshold function:

$$
\mathbf{u}_{i} \leftarrow \operatorname{soft}\left(\mathbf{a}_{i}+\mathbf{d}_{i}, 0.5 \lambda_{i} / \mu_{i}\right), \quad i=1,2 .
$$

To find the solution to ( $7 \mathrm{~b})$ in explicit form, note that the solution to

$$
\begin{array}{cl}
\underset{\mathbf{a}_{1}, \mathbf{a}_{2}}{\operatorname{argmin}} & \mu_{1}\left\|\mathbf{b}_{1}-\mathbf{a}_{1}\right\|_{2}^{2}+\mu_{2}\left\|\mathbf{b}_{2}-\mathbf{a}_{2}\right\|_{2}^{2} \\
\text { such that } & \mathbf{x}=\Phi_{1}^{*} \mathbf{a}_{1}+\Phi_{2}^{*} \mathbf{a}_{2}
\end{array}
$$

is given by

$$
\mathbf{a}_{i}=\mathbf{b}_{i}+\frac{1}{\mu_{i}}\left(\frac{1}{\mu_{1}}+\frac{1}{\mu_{2}}\right)^{-1} \Phi_{i}\left(\mathbf{x}-\Phi_{1}^{*} \mathbf{b}_{1}-\Phi_{2}^{*} \mathbf{b}_{2}\right)
$$

for $i=1,2$, where (5) has been used. Therefore the body of algorithm (7) can be written as

$$
\begin{aligned}
\mathbf{u}_{i} & \leftarrow \operatorname{soft}\left(\mathbf{a}_{i}+\mathbf{d}_{i}, 0.5 \lambda_{i} / \mu_{i}\right) \\
\mathbf{g} & \leftarrow \mathbf{x}-\Phi_{1}^{*}\left(\mathbf{u}_{1}-\mathbf{d}_{1}\right)-\Phi_{2}^{*}\left(\mathbf{u}_{2}-\mathbf{d}_{2}\right) \\
\mathbf{a}_{i} & \leftarrow\left(\mathbf{u}_{i}-\mathbf{d}_{i}\right)+\frac{1}{\mu_{i}}\left(\frac{1}{\mu_{1}}+\frac{1}{\mu_{2}}\right)^{-1} \Phi_{i} \mathbf{g} \\
\mathbf{d}_{i} & \leftarrow \mathbf{d}_{i}-\left(\mathbf{u}_{i}-\mathbf{a}_{i}\right) .
\end{aligned}
$$

A slight simplification is obtained by a change of variables. Defining $\mathbf{v}_{i}=\mathbf{u}_{i}-\mathbf{d}_{i}$, gives the algorithm:

$$
\begin{aligned}
\mathbf{v}_{i} & \leftarrow \operatorname{soft}\left(\mathbf{a}_{i}+\mathbf{d}_{i}, 0.5 \lambda_{i} / \mu_{i}\right)-\mathbf{d}_{i} \\
\mathbf{g} & \leftarrow \mathbf{x}-\Phi_{1}^{*} \mathbf{v}_{1}-\Phi_{2}^{*} \mathbf{v}_{2} \\
\mathbf{a}_{i} & \leftarrow \mathbf{v}_{i}+\frac{1}{\mu_{i}}\left(\frac{1}{\mu_{1}}+\frac{1}{\mu_{2}}\right)^{-1} \Phi_{i} \mathbf{g} \\
\mathbf{d}_{i} & \leftarrow \mathbf{a}_{i}-\mathbf{v}_{i} .
\end{aligned}
$$

A slight rearrangement eliminates redundant computations:

$$
\begin{aligned}
& \mathbf{v}_{i} \leftarrow \operatorname{soft}\left(\mathbf{a}_{i}+\mathbf{d}_{i}, 0.5 \lambda_{i} / \mu_{i}\right)-\mathbf{d}_{i} \\
& \mathbf{g} \leftarrow \mathbf{x}-\Phi_{1}^{*} \mathbf{v}_{1}-\Phi_{2}^{*} \mathbf{v}_{2} \\
& \mathbf{d}_{i} \leftarrow \frac{1}{\mu_{i}}\left(\frac{1}{\mu_{1}}+\frac{1}{\mu_{2}}\right)^{-1} \Phi_{i} \mathbf{g} \\
& \mathbf{a}_{i} \leftarrow \mathbf{d}_{i}+\mathbf{v}_{i}, \\
& \text { go to (11a), }
\end{aligned}
$$

where $i=1,2$, for each line of the algorithm. For initialization: $\mathbf{d}_{i}$ can be initialized to the all-zero vector, with the size of $\mathbf{d}_{i}$ being the same as $\mathbf{a}_{i}$. The vectors $\mathbf{a}_{i}$ can also be initialized to zero because the algorithm converges regardless. However it makes sense to initialize using $\mathbf{a}_{i}=0.5 \Phi_{i} \mathbf{x}$ as this satisfies (4b).

\section{References}

[1] M. V. Afonso, J. M. Bioucas-Dias, and M. A. T. Figueiredo. Fast image recovery using variable splitting and constrained optimization. IEEE Trans. Image Process., 19(9):23452356, September 2010.

[2] S. Boyd, N. Parikh, E. Chu, B. Peleato, and J. Eckstein. Distributed optimization and statistical learning via the alternating direction method of multipliers. Foundations and Trends in Machine Learning, 3(1):1-122, 2011.

[3] L. Daudet and B. Torrésani. Hybrid representations for audiophonic signal encoding. Signal Processing, 82(11):15951617, November 2002.

[4] M. E. Davies and L. Daudet. Sparse audio representations using the MCLT. Signal Processing, 86(3):457-470, March 2006 .

[5] M. J. Fadili, J.-L. Starck, J. Bobin, and Y. Moudden. Image decomposition and separation using sparse representations: An overview. Proc. IEEE, 98(6):983 -994, June 2010.

[6] B. Himed, editor. Over-The Horizon Radar Technology Risk Reduction Initiative, Phase I Final Report. RF Technology Branch, Sensors Directorate, AFRL.

[7] I. W. Selesnick. Resonance-based signal decomposition: A new sparsity-enabled signal analysis method. Signal Processing, 91(12):2793 - 2809, 2011.

[8] I. W. Selesnick. Sparse signal representations using the tunable Q-factor wavelet transform. In Proceedings of SPIE, volume 8138 (Wavelets and Sparsity XIV), 2011.

[9] I. W. Selesnick and I. Bayram. Oscillatory + transient signal decomposition using overcomplete rational-dilation wavelet transforms. In Proceedings of SPIE, volume 7446 (Wavelets XIII), 2009.

[10] J.-L. Starck, M. Elad, and D. Donoho. Image decomposition via the combination of sparse representation and a variational approach. IEEE Trans. Image Process., 14(10):15701582 , October 2005 